\title{
Activin/myostatin receptor signaling and vascular calcifications in chronic kidney disease: A "liaison dangereuse"?
}

\author{
Giacomo Garibotto $^{1,2(1)}$, Pasquale Esposito ${ }^{1,2}$, Daniela Picciotto ${ }^{1,2}$, Daniela Verzola ${ }^{1,2}$ \\ 'Division of Nephrology, Dialysis and Transplantation, University of Genova, Genova, Italy \\ ${ }^{2}$ Department of Internal Medicine, IRCCS Ospedale Policlinico San Martino, Genova, Italy
}

Both myostatin (MSTN) and activin A, two peptide members of the transforming growth factor (TGF)- $\beta$ super family, have been suggested to play major roles in complications of chronic kidney disease (CKD), including vascular and bone disease. Both MSTN and activin A share many similarities in terms of structure, signaling pathway, and functions with TGF- $\beta$, and have been initially studied as players in muscle cachexia in CKD and several other chronic diseases [1]. However, the last few years have witnessed a paradigm shift with respect to our understanding of the effects of MSTN/activin signaling in organs distant from muscle. There is ever-increasing evidence that the MSTN/activin pathway impacts the heart, arterial vessels, insulin sensitivity and vascular remodeling [2]. In addition, recent observations strongly suggest that activin A signaling plays a major role in the progression of kidney disease and $\mathrm{CKD} /$ mineral bone disorder (MBD) [2]. These "off target" actions of MSTN and activin A might contribute substantially to the pathophysiology of wasting, inflammation, vascular damage, and possibly

\footnotetext{
Received August 6, 2019; Revised August 9, 2019;

Accepted August 9, 2019

Edited by Gheun-Ho Kim, Hanyang University, Seoul, Republic of Korea Correspondence: Giacomo Garibotto

Department of Internal Medicine, Division of Nephrology, Dialysis and Transplantation, Università di Genova, Viale Benedetto XV, 6, 16132 Genoa, Italy. E-mail: gari@unige.it

Copyright ( 2019 by The Korean Society of Nephrology

(a) This is an open-access article distributed under the terms of the Creative Commons Attribution Non-Commercial License (http://creativecommons. org/licenses/by-nc-nd/4.0/), which permits unrestricted non-commercial use, distribution, and reproduction in any medium, provided the original work is properly cited.
}

progressive renal dysfunction in CKD.

Like other members of the TGF- $\beta$ family, MSTN is synthesized as a precursor protein made up of two protein domains, an NH2-terminal pro-peptide and a mature $\mathrm{COOH}$-terminus domain, which after cleavage, give rise to a $26-\mathrm{kDa}$ active processed peptide $[1,2]$. Of special interest is the fact that in muscle, activin A and MSTN bind to the same surface receptor complex and activate the same signaling cascade that leads to Smad2/3 translocation into the nucleus (Fig. 1). Binding of MSTN and activin to the ActRIIB and ActRIIA receptor complexes, respectively, on cell membranes leads to activation of Smad2/3-mediated transcription, which in turn stimulates protein breakdown, inhibits protein synthesis by suppressing Akt signaling, and promotes tissue fibrosis [1]. MSTN binds with a weaker affinity to the ActRIIA receptor than to the ActRIIB receptor. The activities of both MSTN and activin A are regulated by their latent complexes, where propeptide remains associated and prevents binding to the receptor, and also by follistatin (FSTL) and FSTL-like proteins, which inhibit activity. Several activities of other members of the TGF- $\beta$ family, such as growth differentiation factor (GDF) 11 , are redundant with those of MSTN and Activin.

ActRIIA/B receptor activation in nonclassical tissues, such as smooth muscle cells, inflammatory cells, and bone may have deleterious effects on organ structure and function (Fig. 1). Besides its actions on protein catabolism, MSTN exerts many effects on glucose metabolism and might participate in the pathophysiology of atherosclerosis. MSTN deficiency protects against insulin resistance and atherosclerosis [2]. In human aortic athero- 


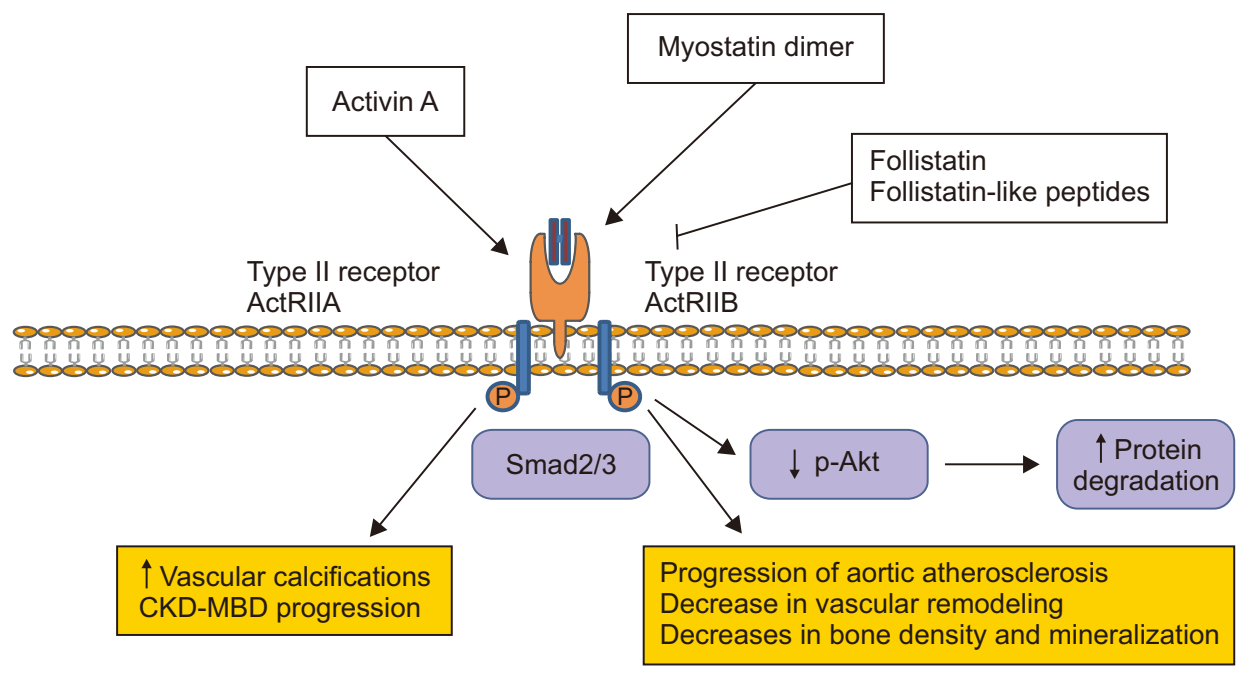

Figure 1. Effects of activin A and myostatin in nonclassical tissues: vascular smooth muscle cells and bone. CKD, chronic kidney disease; MBD, mineral bone disorder. sclerosis, MSTN is upregulated in the media, neointima, and cells infiltrating the vessel wall, where it plays an active role in monocyte chemiotaxis and ultimately in hindering vascular wall remodeling [3]. The effects of MSTN on bone are less well-studied. Studies of MSTN-knockout mice revealed that early bone regeneration and inhibition of MSTN leads to an increase of osteogenesis. $\mathrm{Myo}^{-/-}$mice showed increases in density, strength, and bone mineralization. In clinical studies, inhibition of MSTN has been shown to increase the osteogenic potential and bone mineralization in patients with diabetes mellitus [4].

Activin A signaling appears to be more involved than MSTN signaling in CKD-MBD and vascular calcification. Activation of the ActRIIA receptors can increase vascular smooth muscle cell (VSMC) transformation into osteoblast-like cells that can cause medial calcifications. Activin A receptor type I/activin-like kinase 2-activating mutations cause fibrodysplasia ossificans progressive (FOP), a genetic disorder leading to heterotopic ossification. Recent data from Hruska et al [5] support the role of activin and its receptor as a cause of vascular disease in CKD. In VSMCs from nondiabetic/nonatherosclerotic aortas, CKD increases both ActRIIA signaling and vascular fibroblast signaling, thus causing osteogenic transition and stimulating vascular calcification [5]. ActRIIA signaling was activated by CKD throughout the body in a murine homologue of X-linked Alport syndrome [6]. In addition, an ActRIIA ligand trap was shown to reverse CKD-stimulated bone remodeling, likely through inhibition of activin-A induced osteoclastogenesis [7]. These observations in preclinical studies identify ActRIIA sig- naling as a therapeutic target for CKD-MBD.

In a study of the association between serum MSTN levels and abdominal aortic calcification (AAC) reported in this issue of Kidney Research and Clinical Practice, Lee et al [8] tested the hypothesis that MSTN is involved in the calcification of the vascular wall in patients with CKD. In a cross-sectional study, they evaluated AAC in the lateral lumbar spine using plain radiography and bone mineral density (BMD) in 71 maintenance hemodialysis patients. They classified patients into two groups according to the median value of MSTN. They observed that higher AAC scores are associated with low MSTN levels. In addition, circulating MSTN had a positive association with skeletal muscle mass and indexes of BMD measured at the total hip and femur neck. Their study, albeit limited by a relatively small number of subjects, is interesting since it identifies the activation of the ActR II receptor as a mediator of vascular damage in CKD.

The emergence of MSTN as a potentially modifiable risk factor in many catabolic conditions has led to growing interest in its measurement in serum as a tool to assess patient risk and target therapy. Muscle MSTN is robustly associated with wasting and muscle atrophy in patients with CKD, but the clinical utility of MSTN measurements in serum or blood is still unknown. The current hypothesis regarding MSTN activation in serum suggests that the latent MSTN complex is secreted into circulation from muscle, and that the activity of this complex is regulated by bone morphogenic protein-1 (BMP-1)/tolloid metalloproteinases and secreted inhibitors [1]. Although complete loss of MSTN expression can induce significant 
muscle fiber hypertrophy, quantitative relationships between MSTN levels and the sizes of mature muscles have not been demonstrated. Muscle mass in mature mice increases in proportion to the extent of MSTN depletion below a certain threshold, but does not increase in mice with $40 \%$ decreases in MSTN expression. These data are also consistent with the lack of correlation between circulating MSTN levels and lean body mass in normal human males [9]. The regulation of mature plasma MSTN production is also poorly known. After cleavage from the promyostatin, most of the mature MSTN is stored in a latent complex in the extracellular space [1], where it is bound to the propeptide that prevents it from binding to the receptor [1]. Activation of the latent complex releases the mature MSTN and allows it to interact with its receptor. Several factors including low $\mathrm{pH}$, free radicals, and proteases have been shown to activate the latent complex [1]. However, the precise mechanisms that regulate MSTN release from its latent complex under physiological and pathological conditions remain a matter for further investigation.

Even less is known about patients with CKD. Plasma MSTN levels are elevated in early stages of CKD and inversely correlated with estimated glomerular filtration rate, suggesting that impaired renal removal and/or increased MSTN production is responsible for increased levels [2]. Activin A plasma levels are also augmented in experimental uremia [5]. However, plasma follistatin-like 3 (FSTL3), which counteracts activin/MSTN signaling, also increases during renal dysfunction [2]. On the contrary, FSTL levels are normally regulated in patients with advanced CKD, but their levels are correlated with inflammation and reduced muscle strength, suggesting the involvement of a mechanism that includes FSTL in the uremic wasting process [2]. In addition, in atherosclerotic lesions MSTN is largely expressed in CD45+ leucocytes infiltrating the vascular wall, suggesting that vascular, but not circulating MSTN, expresses atherosclerosis progression [3].

There are several caveats in the interpretation of the results obtained by Lee et al [8]. One is that the CKD subjects studied had circulating MSTN levels within the normal range. The biological meaning of variations in plasma MSTN within the physiological range, as observed in this study, is not known. Lee et al [8] hypothesize that lower MSTN levels reflect decreased muscle mass. As a matter of fact, in genetic diseases leading to muscle dystrophy (but not in healthy subjects), preserved muscle mass is associated with greater MSTN plasma levels, since MSTN is produced within skeletal muscle [10]. Another puzzling issue is that in patients with CKD, there are several other circulating molecules that increase in the blood and may act on the activin II A/B receptor, such as activin A, GDF 11, and FSTL. In particular, plasma FSTL3, which counteracts activin/MSTN signaling, increases in renal dysfunction, and its levels are correlated with inflammation and reduced muscle strength [11]. Clearly, obtaining measures of plasma MSTN and its circulating inhibitors in blood could help assess the utility of measuring plasma MSTN as a biomarker of wasting.

In addition, there remain analytical and clinical challenges in translating MSTN serum testing into routine practice. Circulating MSTN levels have been measured by enzyme-linked immunosorbent assay (ELISA)-based assays directed at mature MSTN growth factor [12]. These assays, however, require acid dissociation of MSTN from the latent complex, with latent MSTN levels calculated based on differences between acid-treated and non-acidtreated serum. Furthermore, the high amino acid similarity of MSTN, activin A, and GDF11 raises the possibility of cross-reactivity in ELISA-based methods of detection. For these reasons, specific MSTN measurements have thus far been achieved only by mass spectrometry-based methods [13].

What is the meaning of the negative association between serum MSTN and AAC? Lee et al [8] suggest that higher MSTN is protective against vascular calcification. As an alternative explanation, the negative association between MSTN and AAC may be an effect of "reverse epidemiology," a concept suggesting that nutritional and metabolic factors associated with worse outcomes in the general population also relate to outcomes in CKD patients, but in an opposite direction. This suggestion is fascinating, but it remains important to consider that previous studies in vascular smooth cell biology have shown that MSTN is primarily implicated in downregulating cell cytoskeleton proteins, and therefore in hindering vascular remodeling, but not in mechanisms of vascular calcification. On the contrary, activin A antagonism may protect against vascular calcifications. Therefore, the association observed may not be causal, and activin A levels (which were not studied by Lee et al [8]) could be 
the link to the observed association.

The data shown by Lee et al [8], albeit preliminary in nature, lead to the hypothesis that acting on ActRIIA/B receptor signaling favorably modifies vascular calcifications. However, the results reported by Lee et al [8] are not sufficient to allow the extrapolation of MSTN measurements into diagnostic strategies for patients with CKD. Given the negative correlation observed between MSTN levels and vascular calcifications and methodologic limitations, the hypothesis that MSTN protects against calcifications needs to be further tested. In addition, studies with larger sample sizes are required to evaluate relationships between circulating MSTN, systemic markers of inflammation, other metabolic parameters, and clinical outcomes, particularly cardiovascular outcomes, in patients with CKD. The tempting results demonstrated by Lee et al [8] are to be considered preliminary with regard to unraveling the mechanisms leading to vascular damage in patients with CKD.

\section{Conflicts of interest}

All authors have no conflicts of interest to declare.

\section{Authors' contributions}

Giacomo Garibotto and Daniela Verzola wrote the manuscript, Pasquale Esposito, and Daniela Picciotto contributed to the discussion and edited the manuscript. All authors read and approved the final manuscript.

\section{References}

[1] Han HQ, Zhou X, Mitch WE, Goldberg AL. Myostatin/activin pathway antagonism: molecular basis and therapeutic potential. Int J Biochem Cell Biol 2013;45:2333-2347.

[2] Verzola D, Barisione C, Picciotto D, Garibotto G, Koppe L. Emerging role of myostatin and its inhibition in the setting of chronic kidney disease. Kidney Int 2019;95:506-517.

[3] Verzola D, Milanesi S, Bertolotto M, et al. Myostatin me- diates abdominal aortic atherosclerosis progression by inducing vascular smooth muscle cell dysfunction and monocyte recruitment. Sci Rep 2017;7:46362.

[4] Wallner C, Jaurich H, Wagner JM, et al. Inhibition of GDF8 (Myostatin) accelerates bone regeneration in diabetes mellitus type 2. Sci Rep 2017;7:9878.

[5] Hruska KA, Sugatani T, Agapova O, Fang Y. The chronic kidney disease-Mineral bone disorder (CKD-MBD): advances in pathophysiology. Bone 2017;100:80-86.

[6] Williams MJ, Sugatani T, Agapova OA, et al. The activin receptor is stimulated in the skeleton, vasculature, heart, and kidney during chronic kidney disease. Kidney Int 2018;93: 147-158.

[7] Sugatani T, Agapova OA, Fang Y, et al. Ligand trap of the activin receptor type IIA inhibits osteoclast stimulation of bone remodeling in diabetic mice with chronic kidney disease. Kidney Int 2017;91:86-95.

[8] Lee SM, Kim SE, Lee JY, Jeong HJ, Son YK, An WS. Serum myostatin levels are associated with abdominal aortic calcification in dialysis patients. Kidney Res Clin Pract 2019;38:481-489.

[9] Lakshman KM, Bhasin S, Corcoran C, et al. Measurement of myostatin concentrations in human serum: Circulating concentrations in young and older men and effects of testosterone administration. Mol Cell Endocrinol 2009;302:2632.

[10] Burch PM, Pogoryelova O, Palandra J, et al. Reduced serum myostatin concentrations associated with genetic muscle disease progression. J Neurol 2017;264:541-553.

[11] Kralisch S, Hoffmann A, Klöting N, et al. FSTL3 is increased in renal dysfunction. Nephrol Dial Transplant 2017;32: 1637-1644.

[12] Han DS, Chen YM, Lin SY, et al. Serum myostatin levels and grip strength in normal subjects and patients on maintenance haemodialysis. Clin Endocrinol (Oxf) 2011;75:857863.

[13] Peiris HN, Ashman K, Vaswani K, Kvaskoff D, Rice GE, Mitchell MD. Method development for the detection of human myostatin by high-resolution and targeted mass spectrometry. J Proteome Res 2014;13:3802-3809. 